\title{
Effects of acute exposure to ultrahigh radiofrequency radiation on three antenna engineers
}

\author{
Christopher J Schilling
}

\begin{abstract}
Three men were accidentally exposed to high levels of ultrahigh frequency radiofrequency radiation $(785 \mathrm{MHz}$ mean frequency) while working on a television mast. They experienced an immediate sensation of intense heating of the parts of the body in the electromagnetic field followed by a variety of symptoms and signs which included pain, headache, numbness, and parasthesiae, malaise, diarrhoea, and skin erythema. The most notable problem was that of acute then chronic headache involving the part of the head which was most exposed.
\end{abstract}

(Occup Environ Med 1997;54:281-284)

Keywords: radiofrequency; antenna; microwave

The term radiofrequency when applied to the electromagnetic spectrum covers the frequency range $100 \mathrm{KHz}-300 \mathrm{GHz}$; the term microwave is applied to the frequency range $300 \mathrm{MHz}-300 \mathrm{GHz}$. The range $300 \mathrm{MHz}-$ $3 \mathrm{GHz}$ (wavelengths $1 \mathrm{~m}-10 \mathrm{~cm}$ ) is termed ultrahigh frequency and includes cellular telephones, television broadcasting, and microwave ovens $(2.45 \mathrm{GHz})$. The range $3 \mathrm{GHz}-$ $30 \mathrm{GHz}$ (wavelengths $10 \mathrm{~cm}-1 \mathrm{~cm}$ ) is termed

4 Albion Square C J Schilling

Correspondence to: Dr C J Schilling, Schilling and Schilling, Consultants in Occupational Health, 4 Albion Square, London E8 4ES.

Accepted 20 November 1996 superhigh frequency and includes radar, satellite, and other microwave communication systems.

The effects of ultrahigh frequency and superhigh frequency are primarily those of heating. The rate at which the energy of radiofrequency radiation is absorbed in body tissues is described as the specific absorption rate expressed as Watts per kilogram (W/kg) and depends on the frequency $(\mathrm{Hz})$ and the power density expressed in Watts per square metre $\left(\mathrm{W} / \mathrm{m}^{2}\right)$, which can be described as the power crossing unit area normal to the direction of wave propagation. The deposition of radiofrequency energy in body tissues varies with their absorption characteristics, which depend to a considerable extent on their water content. Tissues such as blood, skin, muscle, brain, and peripheral nerves will absorb much more energy than fat and bone, with the result that much of the incident radiofrequency energy tends to pass through the surface fatty tissue to be deposited in the deeper tissues such as muscle and brain.

The figure shows that based on the absorption characteristics of the human body radiofrequency can be subdivided into four regions. The frequency involved in the exposure of the antenna engineers $(785 \mathrm{MHz})$ is the "hotspot" range which lies between $400 \mathrm{MHz}$ and $2 \mathrm{GHz}$. Considerable localised absorption of energy can be expected at incident power densities of about $100 \mathrm{~W} / \mathrm{m}^{2}$, and at $785 \mathrm{MHz}$ hotspots are likely to be several centimetres in size. The heating is highly nonuniform with typical ratios between spatial peaks and whole body average specific absorbtion rates of the order of $150: 1$ to $200: 1$.

There are few published accounts of acute exposure to radiofrequency. Graham ${ }^{1}$ described the results of incidents of overexposure to radar $(1-10 \mathrm{GHz})$ in 58 United States Air Force personnel. Twenty six felt a warming sensation and all reported headache, nausea, fatigue, malaise, and palpitations. Detailed medical evaluation was carried out including neurological and opthalmological examinations, and serum enzymes and blood counts. No significant abnormalities were detected. Some high level exposures $\left(>500 \mathrm{~mW} / \mathrm{cm}^{2}\right)\left(1 \mathrm{~mW} / \mathrm{cm}^{2}=10 \mathrm{~W} / \mathrm{m}^{2}\right)$ resulted in severe anxiety necessitating short term sedation and admission to hospital.
Variation of normalised specific absorption rate with frequency and related absorption characteristics in living organisms: reproduced with the permission of WHO from: Electromagnetic fields (300 Hz to $300 \mathrm{Ghz}$ ) 1993:76. (Environmental Health Criteria 137.) 
Forman $e t a l^{2}$ reported on two radar operators accidentally exposed in the $x$-band (9$10.9 \mathrm{GHz}$ ). The power density was calculated to have been between $60-90 \mathrm{~mW} / \mathrm{cm}^{2}$.

One man was exposed for 80 seconds and reported severe chest pain, vertigo, and a heating sensation of the chest and head plus facial erythema which persisted for three days. Postprandial stomach cramps, dysphagia, shoulder soreness, and gritty eye sensations occurred during the first day and persisted for several weeks. He also experienced recurrent severe headaches, insomnia, irritability, and emotional lability. The peak severity of his subjective complaints occurred three months after exposure. He still had psychological problems a year after the incident.

The second man was exposed for about 75 seconds. He reported a heating sensation of the head and chest plus a headache. He had erythema of his chest and face lasting a day and complained of irritability, insomnia, headaches, photophobia, and blurred vision. A year after the incident he was reporting emotional lability and insomnia.

Hocking $\mathrm{et} \mathrm{al}^{3}$ reported an accident involving nine radio linemen who were dismantling a television bearer and were exposed to radiofrequency $(4 \cdot 1 \mathrm{GHz})$. The maximum exposures were of two men at levels of $4.9 \mathrm{~mW} / \mathrm{cm}^{2}$ for up to 90 minutes. Comprehensive medical examinations were performed and it was concluded that the exposures had not resulted in harmful effects.

Wayne et $a l^{4}$ described a microwave oven $(2.45 \mathrm{GHz})$ accident resulting in an acute selective radial neuropathy with chronic dysasthesia (a crushing sensation). Nerve conduction studies and electromyography were normal. The dysasthesia was still present after 20 months.

Marchiori et $a l^{5}$ reported a case of acute multiple sensory neuropathy after accidental exposure to oven microwaves $(2 \cdot 45 \mathrm{Ghz})$. A cook had acute exposure of her hands and face with pain and dysasthesia. She developed a glove neuropathy of the right hand and a discrete neuropathy of the fingers of the left hand and a sensory neuropathy involving the right trigeminal nerve. Electromyography showed involvement of both median nerves which was more severe in the right hand.

Kolmodin-Hedmin et $a l^{6}$ showed significantly impaired two point discrimination compared with controls in women who used plastic welding machines and were exposed to radiofrequency at $20-30 \mathrm{MHz}$ with $50 \%$ of power densities exceeding $250 \mathrm{~W} / \mathrm{m}^{2}$

McRee and Wachtel ${ }^{78}$ have shown that changes in the ionic gradient of nerve cell membranes can occur independently of the thermal lesion caused by microwaves and that this may be due to either direct interference with the Na-K-ATPase pump or by overloading it with additional influx of sodium and efflux of potassium.

Case report

Three men (called A, B, and C in the text) were employed to service the main four channel UHF television antenna array. To maintain the service a reserve antenna consisting of 12 Sira 2-wavelength panels in four tiers (mean frequency $785 \mathrm{MHz}$ ) with about $1.75 \mathrm{KW}$ mean power at array input per channel was in use. On 5 June 1993 they were working on the mast well below the reserve antenna when at 1140 am by accident the skip in which they were standing was winched up instead of down, bringing the left side of their head, neck, arm, and trunk into close range of these high power aerials; man B's head was about 3-6 inches from a panel when he bent over to try and use the radio telephone. From the waist down they were shielded by the skip. They had to struggle to free the skip as it caught on the antenna panel and started to tip over as it was winched upwards. If they had not done this they would have fallen out to their almost certain deaths.

They were in the reactive "near field" region of the antenna, in which the electromagnetic wave has not fully formed. Field patterns are complicated in this region and an object or in this case the human body in a near field will disturb it and prevent its accurate measurement. The period of exposure was estimated to have been between 50 seconds and $2.5 \mathrm{~min}$ utes. Their radiation meter registered a full scale of $20 \mathrm{~mW} / \mathrm{cm}^{2}, 100 \mathrm{~mm}$ directly in front of the panel; their exposure is likely to have been substantially more. It has not been possible to estimate accurately the dose received.

All three men experienced an immediate sensation of intense heating to the left side of the head, neck, and left arm, similar to an overexposure to sunlight. This was followed by pain in the left side of the head, the eyelids, left ear, and left side of the face. They were all standing with the left side of their bodies facing the antenna panel. Reddening of the skin of the left side of the face and left ear, similar to sunburn, was noted by doctors examining them about an hour after the incident. The erythema lasted three to four days. All three men experienced headache at the site of maximum heating on the left side of the head, which started about an hour after exposure. Man C's symptoms and signs were less severe than those of men $A$ and $B$. He was further away from the antenna panel than his two colleagues.

Man A (aged 44) had a severe headache for two days, had a burn with blisters on the left side of his tongue adjacent to a gold filling, which became very hot during the period of exposure. He complained of nausea and vomiting which started 24 hours after the incident. He vomited four times over a period of 18 hours. He had diarrhoea which started six hours after the incident, was severe for 12 hours, and lasted for a total of 36 hours. $\mathrm{He}$ complained of numbness and parasthesiae on the left side of his head, face, ear, left shoulder, arm, and forearm (I detected diminished light touch and pin prick) made worse by exposure to the sun or heating. He also had erythema of the left upper arm anteriorly. He had lassitude, lack of stamina, and drowsiness, plus chronic headache in the area of the left side of the head, which was maximally exposed to radiofre- 
quency. These symptoms have gradually improved over the period of three years since the incident but he still has chronic but less severe left sided headaches plus localised dysasthesia in the left frontoparietal region. I first saw man A seven weeks after the incident and have seen him twice for follow up since.

Man B (aged 47) also had an area of erythema on the left upper chest anteriorly. His face felt swollen, with tenderness of the left temple, cheek bone, eyelid, and ear. Twenty four hours after the incident he described his eyes as "feeling like arc eye" which he had experienced many years before. His eyes ached and watered for about a week and then cleared up. Four months after the incident he developed a single solar keratosis of the left ear lobe about 5 $\mathrm{mm}$ in diameter; this is likely to have been the site of maximum exposure to radiofrequency. He had a severe headache lasting six days after the incident and experienced an increase in salivation from the left side of his mouth for three days associated with aching on chewing. He had an area of loss of light touch and pin prick in the area of innervation of the left middle branch of the trigeminal nerve (elicited by me); this gradually improved but three years after the incident he still has a slight feeling of numbness and says the left side of his face feels different from the right. He had diarrhoea which started 36 hours after the incident and lasted for 36 hours but was not severe. He had lassitude, lack of stamina, drowsiness, and chronic left sided frontoparietal headache, which was made worse by exposure to the sun or heating. These symptoms have improved over the past three years but he continues to have headaches. I first saw him three weeks after the incident and have seen him twice since for follow up. Both man A and man B said that it took about a year until they considered that they were improving, particularly from the feelings of lassitude and general malaise.

Man C (57) complained of a feeling of discomfort over the left temple and face made worse by exposure to sun light and heating, which improved one month after the incident but although much better 18 months after the incident he was still aware that there was a difference between the left side of his head and face and the right. I saw him for the first time five months after the incident and have seen him once for follow up.

All three men had full blood pictures and biochemistry performed within 72 hours of the incident, which were repeated a month later. All the results were within the normal range.

All three men are white experienced antenna engineers. This job entails undertaking work on antenna towers of a specialised technical nature rather than of a heavy physical type. It does necessitate being fit to climb several hundreds of feet on a regular basis to get to those parts beyond the reach of lifts and other mechanical devices. All three had worked in this capacity for many years for the same employer, man C being the most senior of the group. They had been required to undergo an annual medical examination to ensure that they were fit to climb which included an examination by a doc- tor, a static ECG, full blood picture, and biochemistry. No notable health problems had ever been detected for any of the men and none had a history of ill health or injury. None of them had histories of mental ill health and none considered that their symptoms were related to the danger they had experienced.

They continue to maintain contact with me and will be followed up as appropriate.

\section{Discussion}

Of the three men exposed, A and B are likely to have had the highest exposures to radiofrequency $(785 \mathrm{MHz})$ which may have been an order of magnitude or possibly considerably more than the National Radiation Protection Board (NRPB) ${ }^{9}$ investigation level for the adult population of $50 \mathrm{~W} / \mathrm{m}^{2}$. When exposures do not exceed these investigation levels, basic restrictions established by the NRPB covering specific absorption rates for various parts of the body are adhered to, with the objective of preventing acute and long term effects.

The heating which occurs as the result of exposure to radiofrequency at $785 \mathrm{MHz}$, is characterised by its rapid rate and uniquely non-uniform spacial pattern of energy deposition. These features make the biological responses to the electromagnetic thermal loading unlike those of other thermal agents. Considerable localised energy absorption can be expected at $785 \mathrm{MHz}$ as this is in the hotspot range. This may explain the intensity of the symptoms and signs experienced. The specific absorption rate is likely to have been so high that the normal avenues of heat transfer of the exposed tissue became inadequate. Burning of the skin occurred and other more deep seated tissue proteins may have been damaged-for example the lining of the bowel resulting in diarrhoea. Damage to sensory nerve endings may account for the symptoms of numbness, dysathesia, and parasthesiae coupled with impairment of light touch and pin prick sensation. The initial severity of the headaches and their persistence has given most cause for concern. The headaches occurred on the side of the head exposed to radiofrequency and were exacerbated by exposure to direct sunlight or body warming. It seems that they may originate from an inflammatory irritative lesion in the peripheral nerves. Their prolonged course may be due to a central component related to facilitation in the reticular formation and its input to the thalamus.

Poor stamina, particularly noticeable when climbing antenna towers has been a major problem particularly for men A and B. Their stamina has gradually improved but not to the levels before this incident.

\section{Conclusion}

Those involved in the incident were fit and active men and the experience has resulted in acute and chronic physical signs and symptoms which have caused considerable distress. This incident underlines the dangers associated with live antenna maintenance and the 
need for the strictest controls to avoid accidental exposures.

1 Graham RB. The medical results of human exposure to radiofrequency radiation. In: The impact of proposed radiofrequency radiation standards on military operations. Neuilly-sur-Seine, France: Advisory Group for Aerospace Research and Development (AGARD) 1985; 6-1-6-8. (Lecture Series No 138.)

2 Forman SA, Holmes CK, McManamon TV, Wedding W/R . Psychological symptoms and intermittent hypertenWR. Psychological symptoms and intermittent hypertension following acute microwave exposure. F Occup Med

3 1982;24:932-4.

Hocking B, Joyner K, Fleming R. Assessment of health
after radio-frequency radiation accident. fournal of Microwave Power and Electromagnetic Energy 1988;23: 67-74.

4 Wayne L, Dickason MD, Barutt JP. Investigation of an active microwave-oven hand injury. 7 Hand Surg 1984; 9A:132-5.

5 Machiori PE, Silva HCA, Hirata MTA, Lino AM, Scaff MJ. Acute multiple mononeuropathy after accidental exposure to oven microwaves. Occup Med 1995;45: 276-7.

6 Kolmodin-Hedman B, Hansson Mild K, Hagberg M, Jonsson E, Andersson M, Eriksson A. Health problems among operators of plastic welding machines and exposure to radiofrequency electromagnetic fields. Int Arch Environ Health 1988;60:243-7.

7 McRee DI, Wachtel H. Pulse microwave effects on nerve vitality. Radiat Res 1982;91:212-8.

8 McRee DI, Wachtel H. Elimination of microwave effects on the vitality of nerves after blockage of active transport. Radiat Res 1986;108:260-8.

9 National Radiation Protection Board. Statement on restrictions on human exposure to static and time varying electromagnetic fields and radiation. Documents of the NRPB 1993;4:5. 